\title{
Analysis and Optimization Performance of the QOS in GSM/EDGE Networks
}

\section{Salih M. Atroshey}

PhD -Electrical and Computer Eng. Dep.

Duhok University. Email:salihatroshey@yahoo.com

\author{
Myasar R. Tabany
}

MSc-Computer Science /Mobile communication. Duhok University. Email:mtabany@yahoo.com

\begin{abstract}
The aim of this paper is to analysis and optimizes the efficiency of the Enhanced Data rates for GSM Evolution (EDGE) Quality of Service (QoS) profilewhich is used for Packet Data Protocol (PDP) context in data transfer over GSM/EDGE networks. Two software tools were used in the practical part: the first one is a Subscriber Management Unit client (SMU/9820) from HUAWEI Technologies Co., Ltd. that is a real time system for mobile network management in HUAWEI Home Location Register (HLR); the second is a DUMETER 5.02, which monitors the network traffic and displays a real time charts. The practical part has been achieved in Asia cell telecommunications Company in three different cities; Mosul, Duhok and Erbil: each one in three levels; the first level is the analysis of available QoS in the network, based on the default profile which depended by the network. However, 24 testing have been recorded; this testing has been repeated for three cities. In second level a hundreds of tests and optimization implemented on tens of QoS and PDP parameters in order to reach better data rate. Final level includes the implementation of the proposed profiles which resulted from second level. Finally, the proposal profiles improved the performance of the QoS which reflected the gains of $34 \%$ in average data rate in the three cities.

Keywords: HLR, GSM, QOS, EDGE.
\end{abstract}

\section{تحليلَ وتُحسينُ كفاعةَ نوعيةِ الخدمةِ (QoS) في شبكات ال GSM/EDGE)}
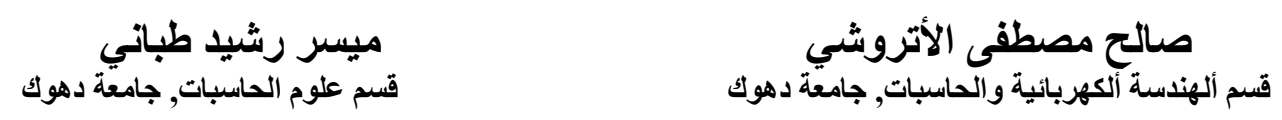

الخلاصة

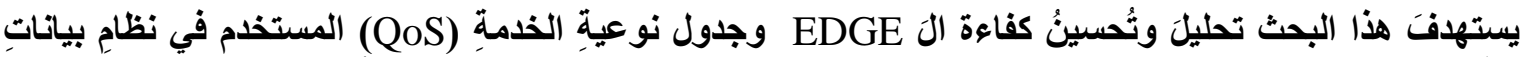

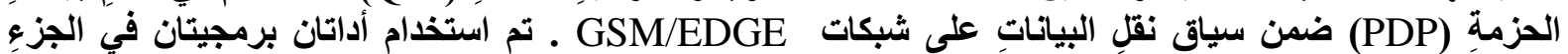
العملي: الأولى وحدةِ إدارةِ المشتركين (SMU/9820) من شركة

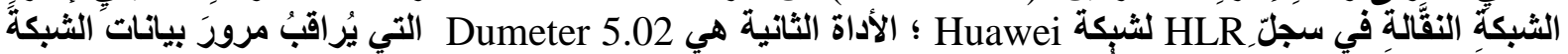

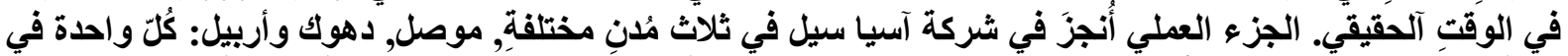

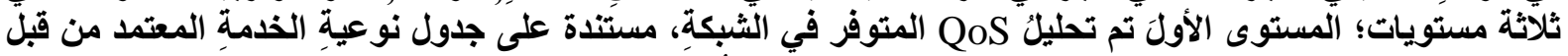

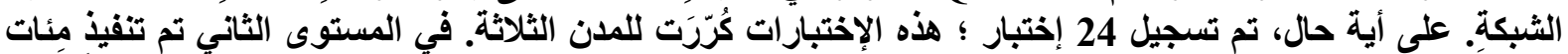

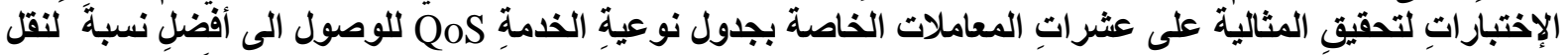

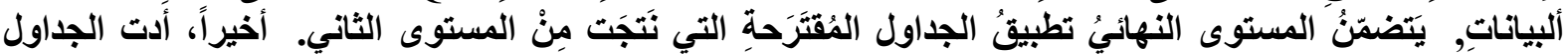

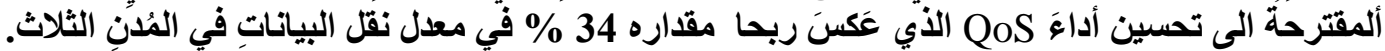




\section{1-Introduction}

The evolution of European mobile communications system toward the Third Generation $(3 \mathrm{G})$ is based on Global System for Mobile communications (GSM). The second step in the evolution is the Enhanced Data rates for GSM Evolution (EDGE). EDGE is a global radio based high speed mobile data standard that can be introduced into GSM/Global Packet Radio Service (GPRS) and IS-136 networks. EDGE allows theoretical data transmission rates up to $384 \mathrm{Kbps}$ in packet switch mode; these throughputs are required to support multimedia services. This is achieved within the same GSM bandwidth and existing frequencies bands. The idea behind EDGE is to increase the data rate that can be achieved in GSM/GPRS by changing the type of modulation used while still working with existing GSM and GPRS network nodes. The new modulation that is introduced is the eight states Phase Shift Keying (8-PSK). EDGE is seen as a second and final step transition from $2 \mathrm{G}$ to $3 \mathrm{G}$, so no new operator licenses are needed for it [8]. The same GPRS profile and PDP parameters such as delay class, reliability class, precedence class, Transfer Handling Priority (THP) and other tens of parameters which is defined in 3GPP (3rd Generation Partnership Project) Release 99(R99) are still used in EDGE [2]. This paper presents three different profiles to provide QoS to EDGE users, and see the differences in transfer rate after implementing each of them. The rest of this paper is organized as follows: section 2 describes an overview of the QoS subscriber profile and its parameters; section 3 presents briefly the software tools used in the practical side; section 4 explain a GSM and EDGE traffic calls and the effects of each one over the other. In section 5 analytical side and testing results are explained and illustrated. Finally, the study is concluded in section 6.

\section{QoS Subscriber Profile and its Parameters}

The performance and capacity gain can be achievable with QoS management in the Packet Switch (PS) radio network [10]. The QoS subscriber profile contains information about the subscriber current connection. When the QoS is implemented, the operator will have to package its parameters in such a way that the subscriber has a simple choice to make rather than be confronted with complex parameters, i.e. the customer cannot be expected to choose a mean throughput class, reliability class, etc. The parameters underlying each service class will be stored in the subscriber profile along with other aspects such as Packet Data Protocol (PDP) address type. The profile is stored in the Home Location Register (HLR) extension known as the GPRS Register (GR) in what is called HLR profile template, many templates can be stored based on different subscription levels and types [11]. The QoS parameters have a central role in the QoS concept; they together form the QoS profile. One QoS profile associated with each PDP context thereby defining the characteristic of the connection through the mobile network, for instance affect the parameters in the radio networks, policing and shaping functions in the Core Network $(\mathrm{CN})$. The need for qualitative and quantitative results for these parameters in a real time situation is critical. One such scenario is the effect on the network performance by means of effective throughput and handoff latency perceived by the mobile user, with increasing number of active users or network load [9]. The QoS profile resides in the HPLMN/HLR that determines which QoS profile to be used in the VPLMN. Furthermore, it is defined per end user and per Access Point Name (APN), i.e. a single end user may have a separate QoS profile for each APN, the actual implementation will therefore vary from vendor to vendor. The first step in assigning QoS occurs when Mobile Station (MS) perform attaches procedure. At this stage it registers its capabilities; radio and networking capabilities are provided in the MS class mark that is stored by the network in the Mobility Management (MM) context for the International Mobile Subscriber Identity (IMSI) so long as the MS is attached. QoS in the complete EDGE network is 
controlled in principle by an Internet Protocol (IP) bearer service manager that resides in a Gateway GPRS Node (GGSN) and potentially also exists in MS [12].The QoS parameter values must be worked in a multi operator environment. To define a QoS contract between MS and the network, PDP contexts containing QoS profiles are negotiated between the MS and the Serving GPRS Support Node (SGSN). The QoS profile Information Element (IE) consists of an IE Identifier (IEI), length field, five fields that contain the values of the service classes, other three fields filled with spare bits (Fig. 1) $[10]$.

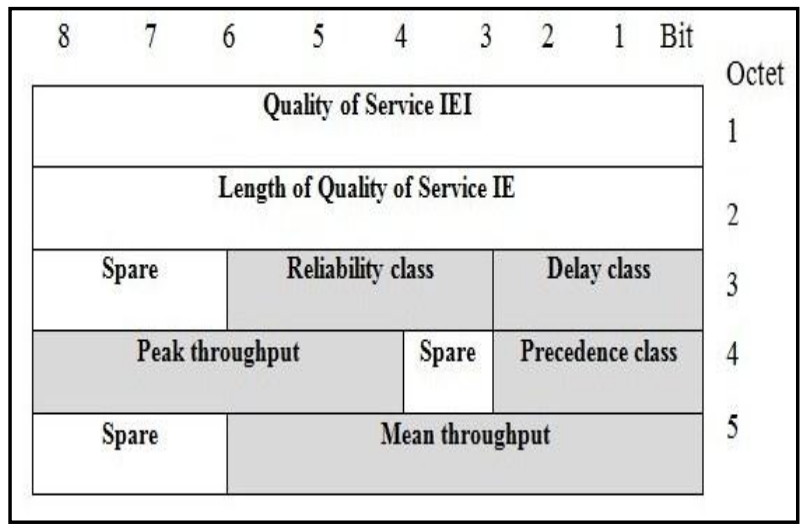

Figure 1: OoS Profile Information

\section{Software Tools}

The HLR SMU/9820 client is the first and main tool used in this paper, it is works in client/server mode to offer user-friendly graphic interfaces, through which subscriber and global data can manage. The SMU server and client communicate through Local Area Network (LAN) or Wide Area Network (WAN). Fig. 2 shows the structure of the SMU client. DUMETER 5.02 is the second tool, which has been used in this paper; it is powerful, user friendly and graphical tool (Fig. 3). It provides an accurate account of the data, which is flowing through the computer network connection at any given moment; furthermore, it generates hourly, daily, weekly and monthly reports. These readouts are presented in both numerical and graphical format. Moreover, DUMETER stopwatch used to accurately measure transfer rates over user specified periods.

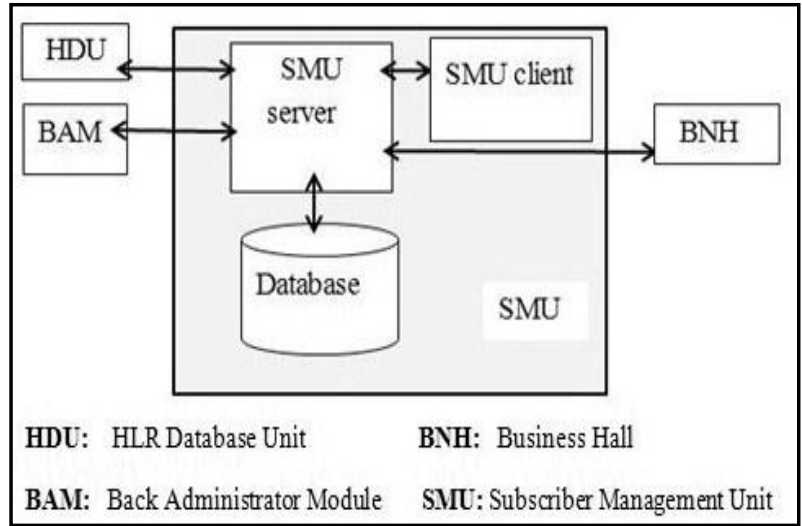

Figure 2 Structure of the SMU Client.

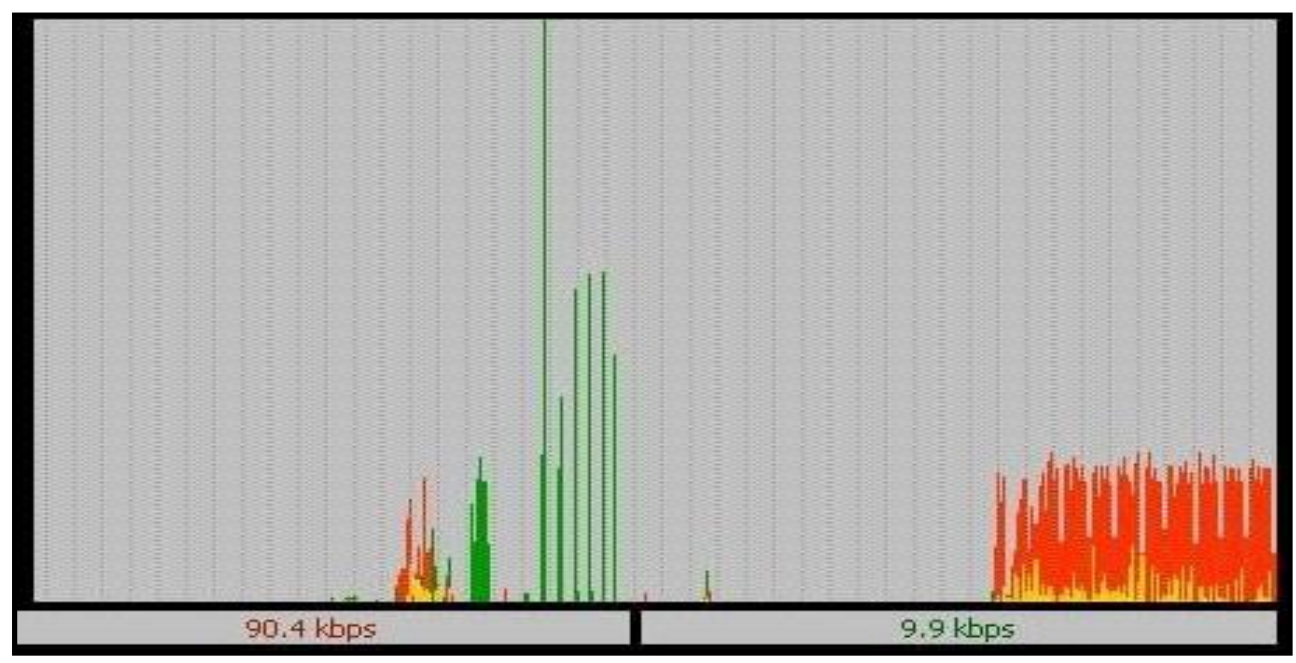

Figure 3 : DUMETER Monitor and Mini Windows. 


\section{GSM/EDGE Traffic Calls and Effects of One over the Other}

EDGE uses the same Radio Resources (RR) and physical channels of the GSM network. Therefore, planning of both GSM and EDGE network is an important issue for GSM network operators. EDGE users send their packets on the idle time slots that are unused by the voice traffic. Although voice users have a higher priority than data users, satisfying data users is very important when it comes to the operating network revenue [5]. For this reason a decision has been taken to obtain the results of the voice traffic at the same times exactly where the EDGE tests have been conducted (Fig.4-A), analysis it to get realistic observation of their effect on data packets transmission in EDGE service for each city. However this process have been doing before and after implementing each one of the new proposed QoS profiles (Fig. 4B), the results shows that there is no significant change occurred on the average calls traffic in the three cities.

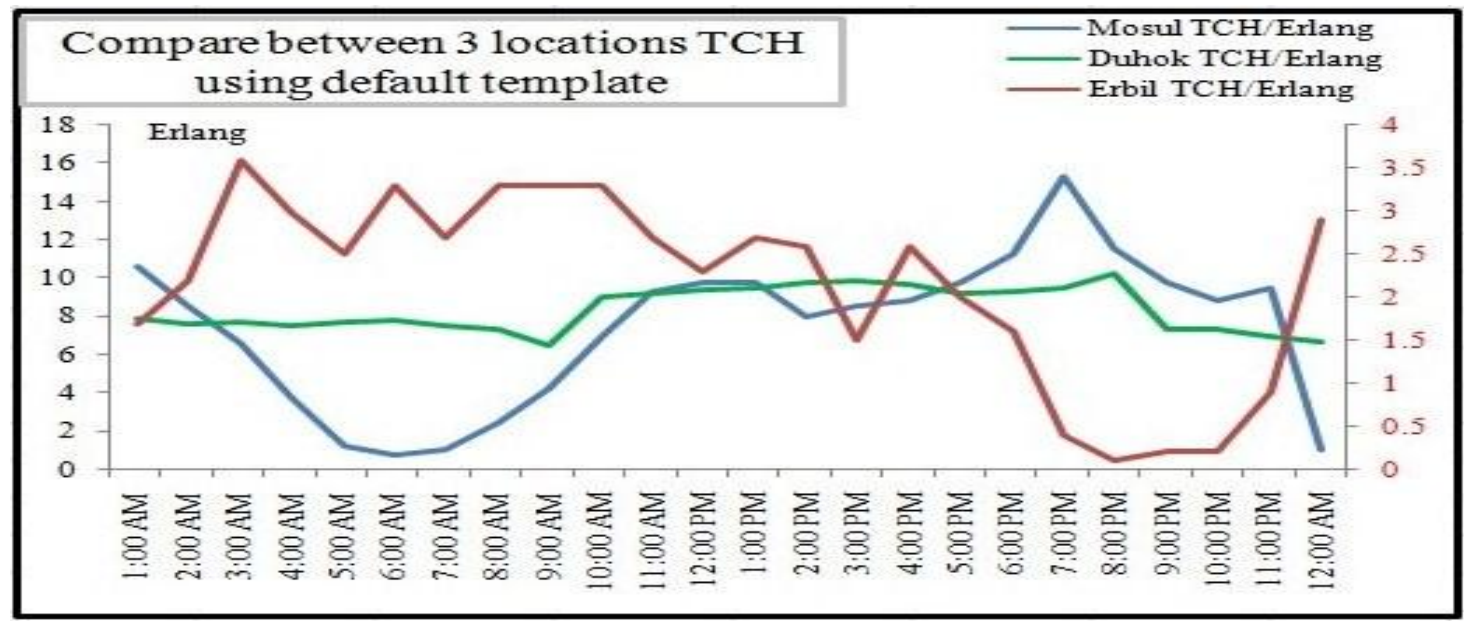

Figure 4-A: Compare between three locations TCH using default template

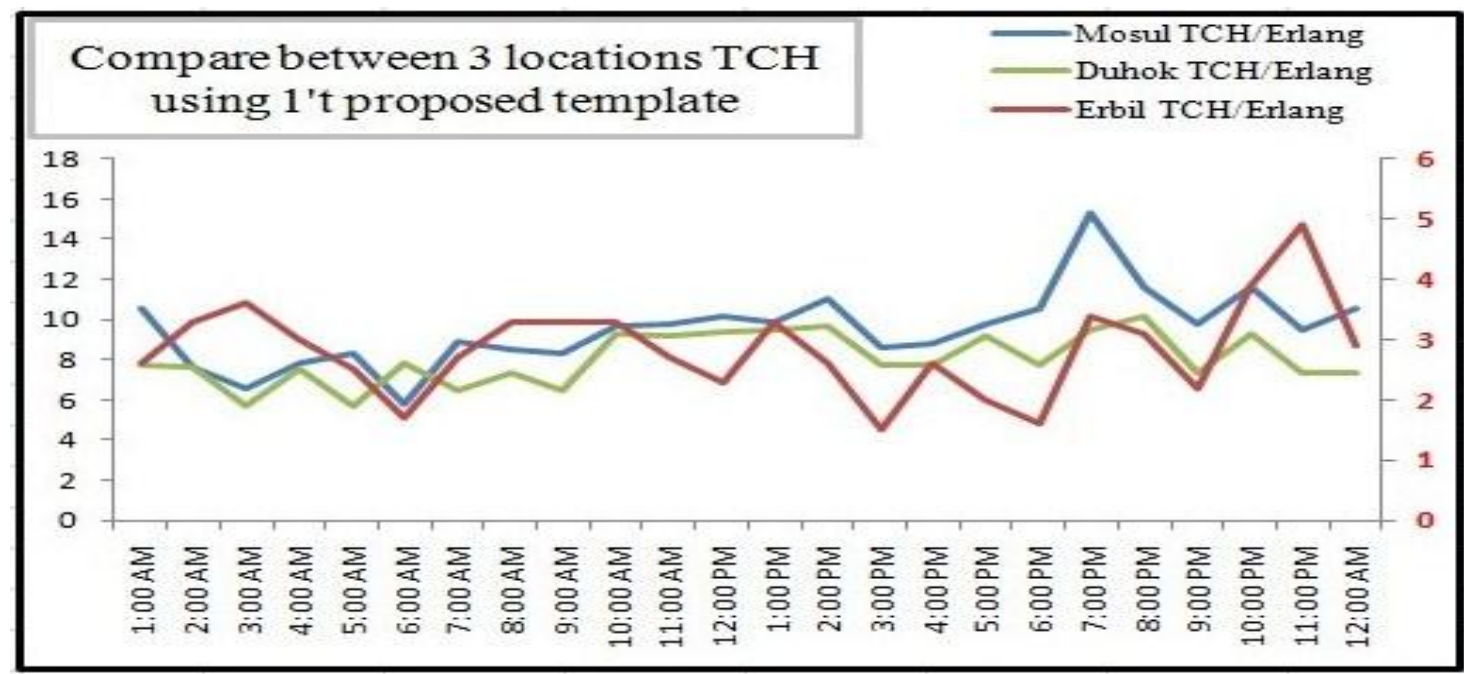

Figure 4-B: Compare between three locations TCH using default template

\section{Analytical Side and Testing Results}

The experimental field started on January 2010, the first step in the process was the connection to the EDGE technology network, so the MS connected to the laptop which has been used as a modem via Bluetooth. The desire file used in the download practical side chosen to be located in one of the standard web sites such as Microsoft.com; The MT used is Nokia N70. Moreover, three different cities have been selected in order to apply the practical part of the paper, Erbil, Duhok and Mosul, with same BTS in each city. 


\subsection{Download part}

The first level is, the analysis part, which was began by conducting a series of tests in the three mentioned cities. The nature of the tests that had by downloaded the desired file from Microsoft.com, then monitor the transfer rate of downloading file in different stages (different percentage) by using DUMETER traffic monitor, up to the end of process, at this moment DUMETER stopwatch import a full report regarding the test include the average transfer rate. The EDGE tests have been conducted 24 times during 24 hours (at the start of each hour), consequently, determine how the traffic calls in each hour effects on the PS network. The second and important level is the optimization level, which includes optimizing tens of QoS profile and PDP context parameters. The optimization of these parameters is crucial to maximizing network performance, generally depends upon BSS physical configuration. Therefore, an effective configuration optimization tool should be used, while taking into consideration network wide influence in future. For most effective, an optimization tool must be capable of ingesting, processing and correlating a wide range of network data from a variety of sources. Therefore, optimization objectives are not so simply stated, it will show the sensitive effects of optimizing connection parameters over the network, in this paper, the hard mission doing through hundreds of tests using active system tool, SMU client. Note that changing operation can accomplish on more than one parameter, but this needs deep understanding of the impact of each parameter alone on the connection characteristic and on the other parameters. Result from this level is proposing two QoS profiles templates compare with the default one (Table-1). The goal from proposing two templates is to give more flexibility to the service provider for dealing with different levels of EDGE subscription and have guaranteed the QoS requirements of the different media (voice, video and data) from the researcher point of view [3], in order to compare different tests based on 72 tests in each city (Figures 8-11).

Table 1: EDGE QoS parameters that have been changed during research

\begin{tabular}{|c|c|c|c|c|}
\hline $\begin{array}{l}\text { Item } \\
\text { No. }\end{array}$ & Name of Parameters & $\begin{array}{l}\text { Default } \\
\text { profile }\end{array}$ & Proposal 1 & Proposal 2 \\
\hline 1 & $\begin{array}{l}\text { GLOBAI CHARGING } \\
\text { CHARACIERIS IIC }\end{array}$ & NONE & NORMAL & NORMAL \\
\hline 2 & RELIABIIITY CLASS & $\begin{array}{l}\text { ACKRL CPR } \\
\text { ODT }\end{array}$ & $\begin{array}{l}\text { UNACKALL } \\
\text { UPRODT }\end{array}$ & $\begin{array}{l}\text { ACKALLP } \\
\text { RODT }\end{array}$ \\
\hline 3 & $\begin{array}{l}\text { IRAFFIC HANDLING } \\
\text { PRIORITY }\end{array}$ & PRIORITY 1 & PRIORITY 2 & $\begin{array}{l}\text { PRIORITY } \\
1\end{array}$ \\
\hline 4 & PRECEDENCE CLASS & NORMAL & NORMAL & HIGH \\
\hline 5 & DELAY CLASS & DELAY 4 & DELAY3 & DELAY I \\
\hline 6 & $\begin{array}{l}\text { AILOCATION/RE TENTION } \\
\text { PRIORITY }\end{array}$ & NORMAL & NORMAL & HIGH \\
\hline 7 & $\begin{array}{l}\text { DEL IVERY OF ERRONEOUS } \\
\text { SDU }\end{array}$ & NODE TECT & YES & YES \\
\hline 8 & MAXTMUM SDU SIZE & $10 \mathrm{OCT}$ & $1500 \mathrm{OCT}$ & $1500 \mathrm{OCT}$ \\
\hline 9 & IR_AFFIC CLASSS & CONVER & INTERACT & BACKGRD \\
\hline 10 & MEAN THROUGHPUT & $\begin{array}{l}500000000 \\
\text { OCT }\end{array}$ & $200000 \mathrm{OCT}$ & $200000 \mathrm{OCT}$ \\
\hline 11 & DELTVERY ORDER & No & YES & YES \\
\hline 12 & RESIDUAAI BII ERROR RAIE & 0.05 & 0.05 & 0.000001 \\
\hline 13 & $\begin{array}{l}\text { AILOW TO ACCESS APN OF } \\
\text { VPLMN }\end{array}$ & FAL CE & TRUE & TRUE \\
\hline 14 & PDP ADDRESS & \multicolumn{3}{|c|}{$\begin{array}{l}\text { PDP ADDRESS INDICATOR is DYNAMIC } \\
\text { so it takes different values det ermined b y the } \\
\text { netw ork operator }\end{array}$} \\
\hline
\end{tabular}




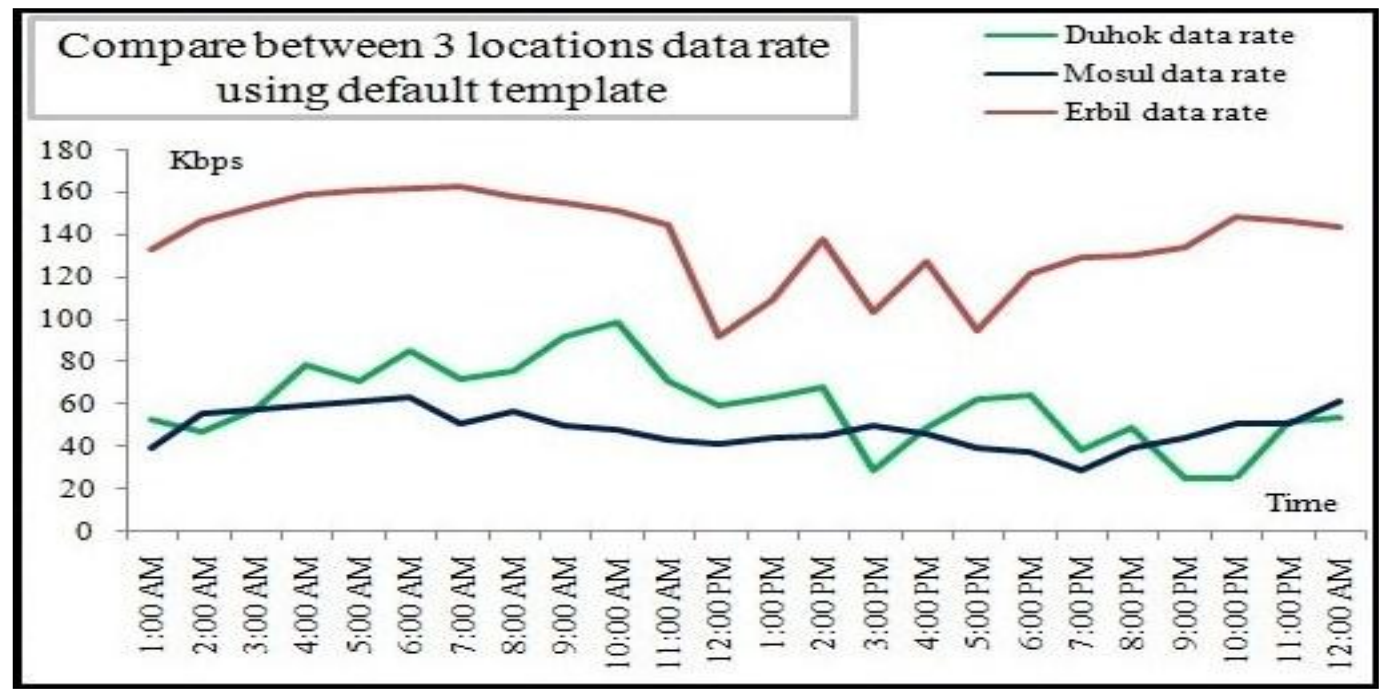

Figure 5-A: Compare between 3 locations data rate using default template

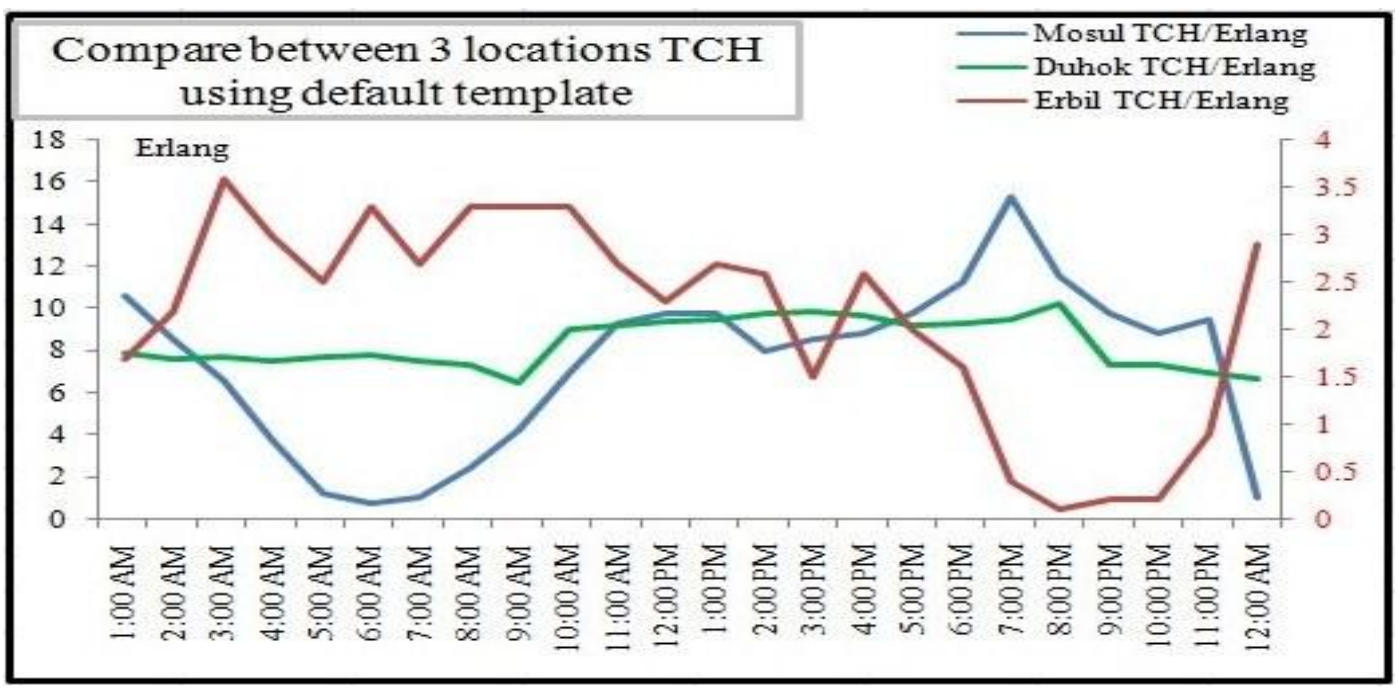

Figure 5-B : Compare between 3 locations traffic channel using default template

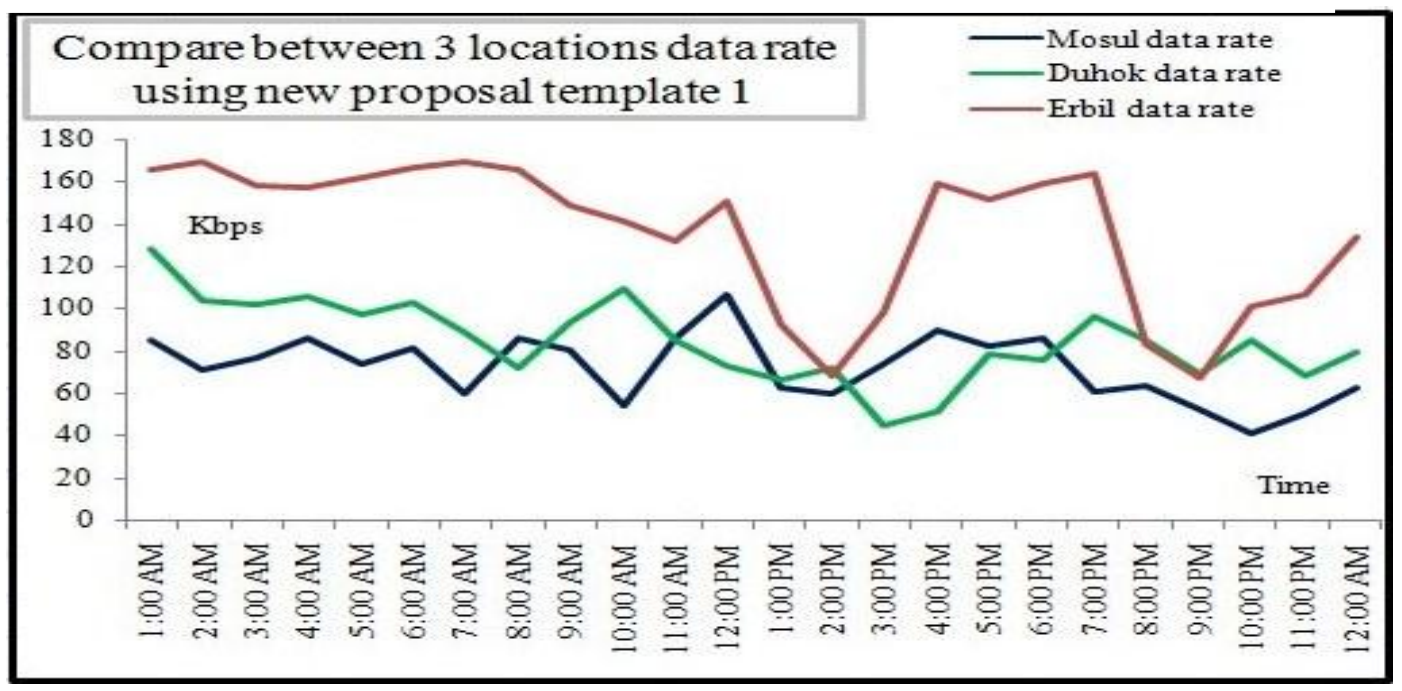

Figure 6 : Compare between 3 locations traffic channel using new proposed template 1 


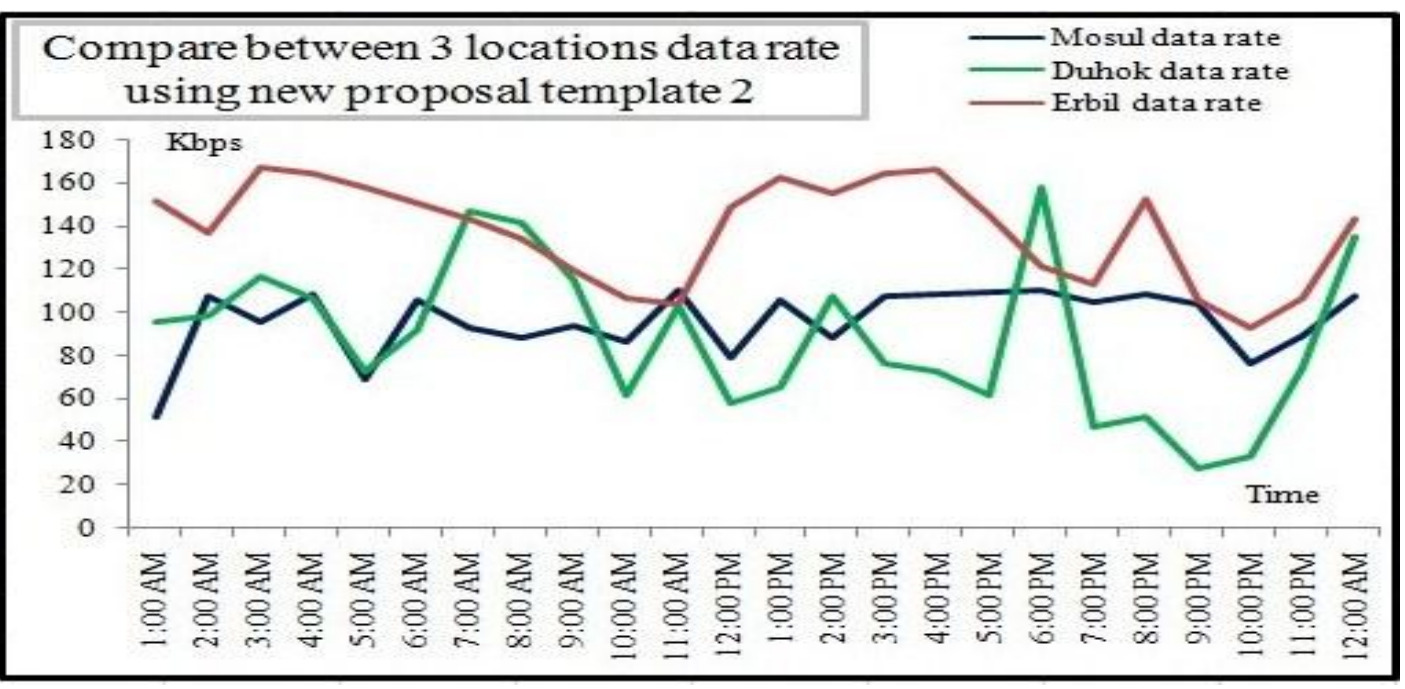

Figure 7 : Compare between 3 locations traffic channel using new proposed template 2

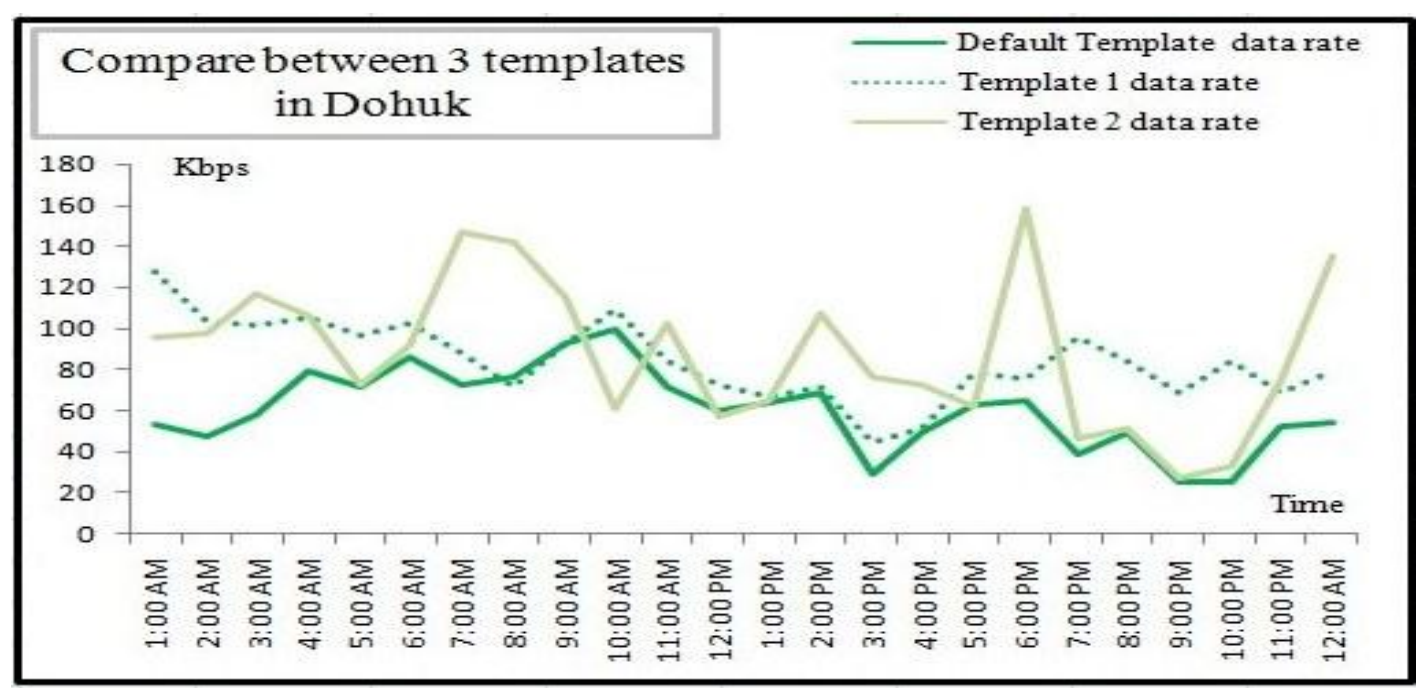

Figure 8: Compare between 3 templates in

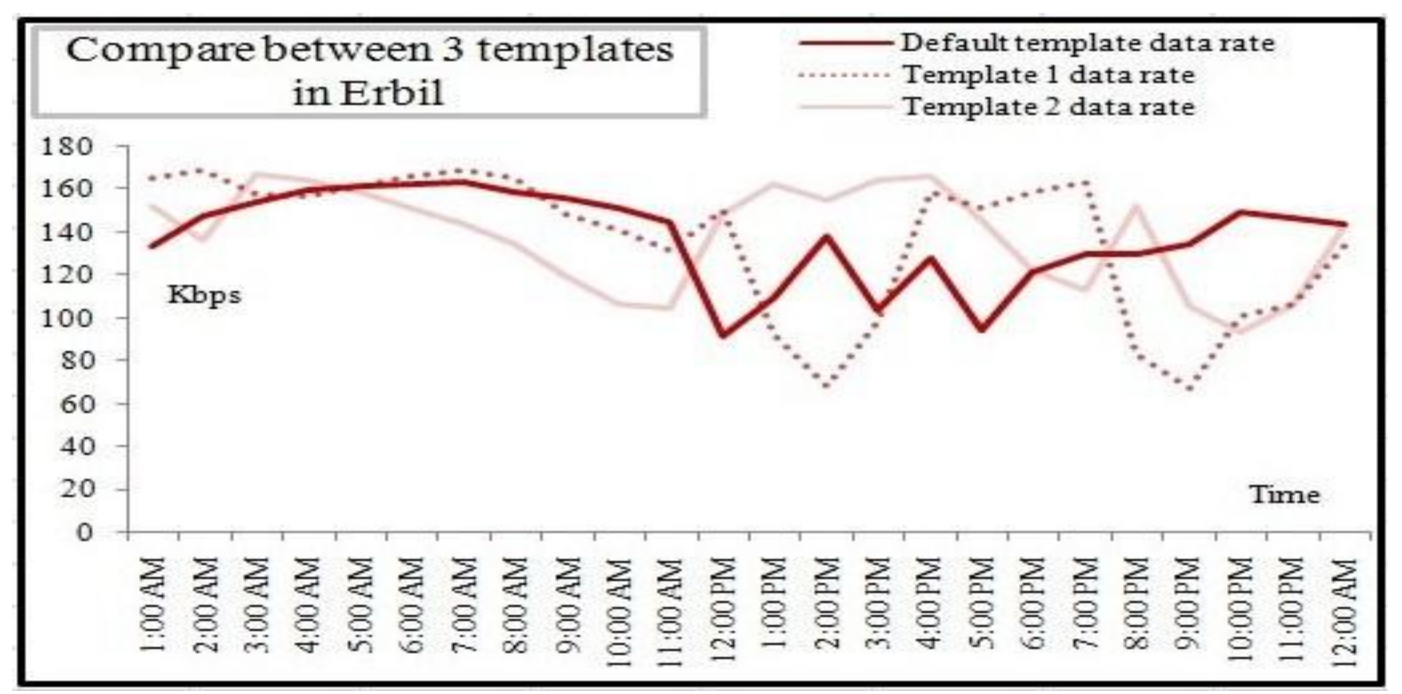

Figure 9: Compare between 3 templates 


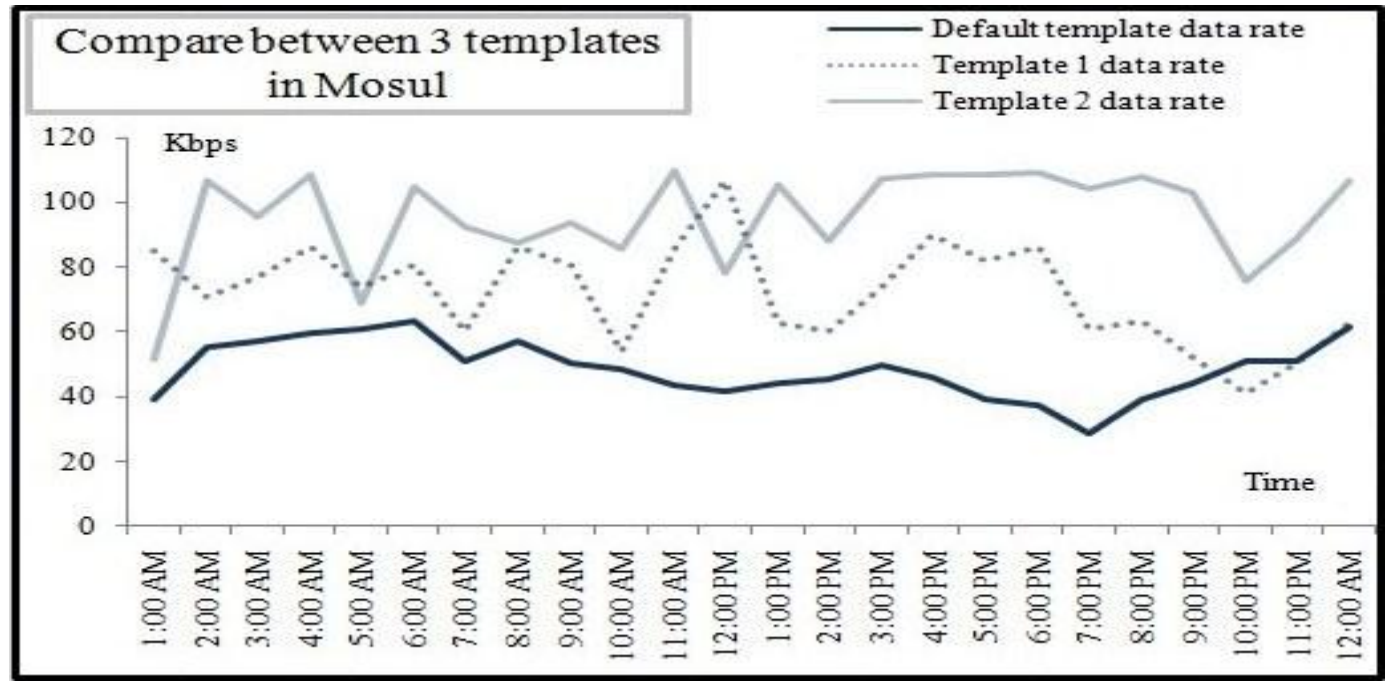

Figure 10: Compare between 3 templates

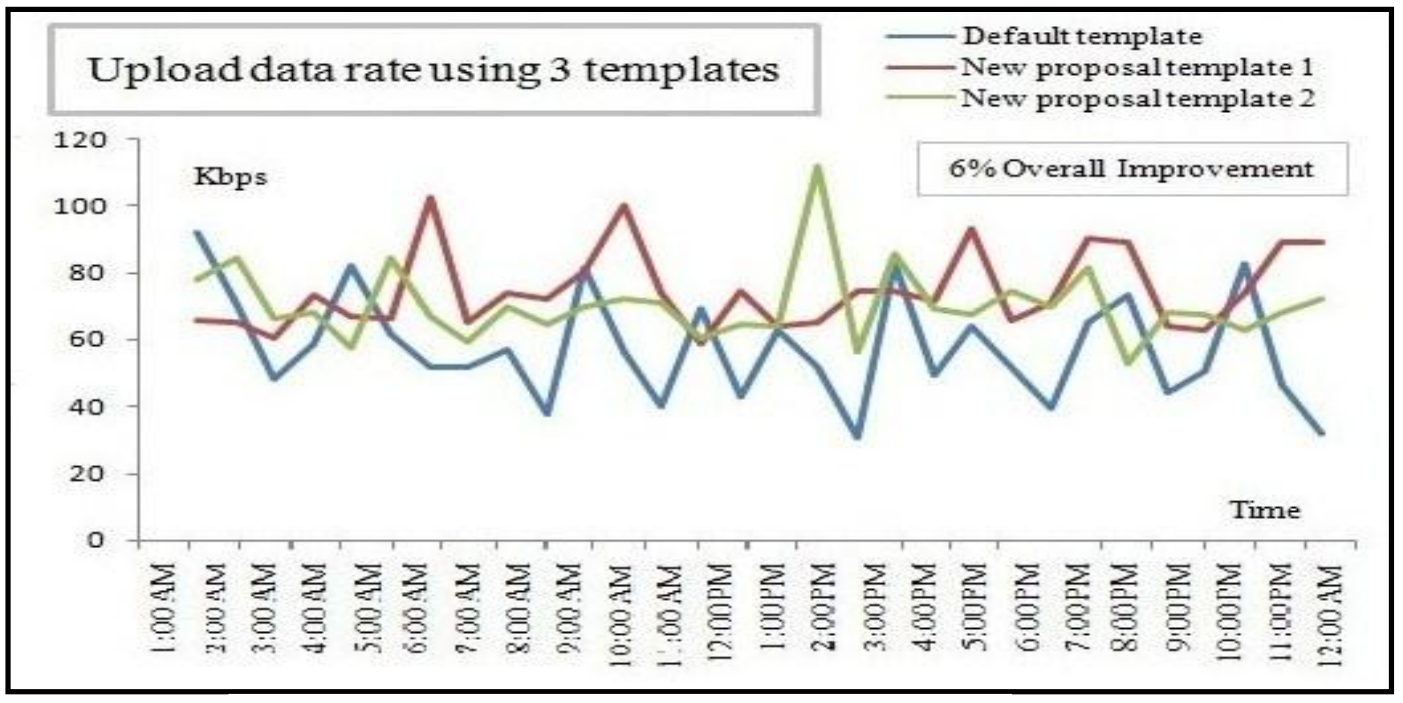

Figure 11: Upload data rate using three

\subsection{Upload part}

So far all which were doing in practical side relate to the download part. In this section, the attempt to highlight the upload data, getting an indicator to its transfer rate will be explained, moreover, whether the QoS profile templates that have been proposed in section 5.1 helped to improve upload service level. However, this section will cover the upload part during handling tests using the three mentioned templates, in one city only. The same file and other tools in the download tests were used in the uploaded tests; 4shared.com was the website which has uploaded the desire file to it. However, average transfer rate that is obtained ranged between $53 \mathrm{Kbps}$ and $65 \mathrm{Kbps}$. It is worth noting that overall percentage improvement at this section was $6 \%$. The results obtained from this section can be illustrated in fig. 11.

\section{Conclusions}

This paper highlighted briefly an overview about GSM/EDGE technology and different proposed solutions to improve its transfer rate. To keep GSM/EDGE competitive, even in years to come, a number of enhancements to improve capacity, coverage, service performance and service continuity have been proposed. All major vendors and many operators have pledged their support for a continued evolution, which, is being standardized 
in 3GPP. However, EDGE transfer rate depends on a number of factors; the most important factor is QoS parameters, which are reside in QoS profile. Analysis and optimization processes, which have been implemented on the parameters based on a real time SMU/9820 client system tool led to deep understand for their impact on the connection characteristics and on other parameters, which ultimately led to proposed two QoS profile templates as. Consequently, many points have been concluded: First, transfer rate before and after the optimization processes has different levels in all three cities, where Erbil has a higher values from others, which is due to many reasons, the most important is calls utilization over them. Second, performance level in EDGE service has been increased generally after implemented the new proposed profiles by more than $27 \%$ for the first profile and more than $42 \%$ for the second profile. Third, the overall improvement ratio in the upload part was less than that achieved in the first download part, taking into consideration that the proposed tests were conducted to a limited extent in only one city. Forth, QoS parameters play a major and direct role in determining the EDGE subscription levels based on their values. And finally the voice calls are ultimately unaffected by the EDGE traffic, on the contrary, where EDGE traffic are too affected by GSM traffic, especially in dealing with the dynamic time slots because of most service providers give voice users higher priority than data users, consequently packet data access may be blocked. However, this unwanted issue can be avoided through configure more time slots as dynamic through TDMA frame.

\section{Acknowledgements}

This work has been supported partially by HUAWEI technologies Co., Ltd. \& Asia cell telecommunications Company; the authors would like to thank the precious help provided by Network Subsystem (NSS) department staff regarding default HLR internet subscriber profile.

\section{References}

[1] Axnas, J., Eriksson, S., Jonsson, T. and Thurfjell, M., "Higher Order Modulation and Turbo Coding for GSM/EDGE Continued Evolution", IEEE vehicular technology conference, Montreal, (September 2006). pp1

[2] Bettstetter, C., Vogel, H. J. and Eberspacher, J., "GSM Phase 2+ General Packet Radio Service GPRS: Architecture, Protocols and Air Interface", IEEE Communications Surveys, volume 2, No. 3, (1999). pp4

[3] Cristina, A., Kochem, B., and Lucia, E., "QoS Provisioning Mechanisms in GPRS Networks", IEEE Sarnoff Symposium on Advances in Wired and Wireless Communications, New Jersey, USA, (March 2003). pp4

[4] GSM Association, "QoS Sensitive Roaming Principles", Ver. 1.0, (August 2004). pp

[5] Lloyd-Evans, R., "QoS in integrated 3G networks", Artech House Publishers, Norwood, MA, (2002). pp

[6] Madkour, M. F., "Effect of high GSM voice traffic on GPRS data network and the proposed solutions", IEEE international symposium on Micro-Nano Mechatronics and Human Science, volume 3, No. 3, (December 2005). pp

[7] Savelli, P., Suree, E. and Pietri, P., "EDGE for mobile Internet", Artech House, Boston, MA, (2001). pp

[8] Stukmann, P., "Quality of Service Management in GPRS Based Radio Access Networks", Kluwer academic publishers, MA, USA, (March 2002). pp

[9] Ylianttila, M., Pande, M., Mäkelä, J. and Mähönen. P., "Optimization Scheme for Mobile Users Performing Vertical Handoffs between IEEE 802.11 and GPRS/EDGE networks", IEEE Global Telecommunications Conference, volume 6, San Antonio, USA, (November 2001). pp1

[10] "MSc Thesis, "Analysis, Optimization and Improvement Performance of the QoS for GPRS Technology in GSM Networks " Duhok University 2010.

The work was carried out at the college of Engineering. University of Duhok 